\title{
Isobaric Vapor-Liquid Equilibrium for Ternary Mixtures of Acetone + Methanol + Ionic Liquids at 101.3kPa
}

\author{
Wenxiu Li, Nana Xu, Honghong Xu, Aidi Zhang, Zhigang Zhang, Tao Zhang * \\ Liaoning Provincial Key Laboratory of Chemical Separation Technology, Shenyang University of Chemical \\ Technology, Shenyang 110142, China
}

* Corresponding author. Tel: +86 024 89383736. E-mail address: 15140056441 @ 163.com (T. Zhang).

\begin{abstract}
In order to avoid the pollution and the corrosion of inorganic anions, such as $\mathrm{BF}_{4}^{-}$, $\mathrm{SO}_{4}{ }^{2-}$ and $\mathrm{Cl}^{-}$, three ionic liquids (ILs) with different organic anions (1-methylimidazolium acetate [MIM]Ac, 1-methylimidazolium 2-hydroxypropionate [MIM]HPr and 1-methyl imidazolium propionate[MIM]Pr) were studied as entrainers for separating acetone + methanol azeotropic mixture. Isobaric vapor-liquid equilibrium (VLE) data for the ternary system containing ILs were measured at $101.3 \mathrm{kPa}$ by using an all-glass dynamic recirculating still. The experimental data are well correlated by using the nonrandom two-liquid (NRTL) model. The separation ability of the three ILs follows the order: $[\mathrm{MIM}] \mathrm{Ac}>[\mathrm{MIM}] \mathrm{HPr}>[\mathrm{MIM}] \mathrm{Pr}$.
\end{abstract}

Keywords: vapor-liquid equilibrium; ionic liquid; acetone; methanol; NRTL model

\section{Introduction}

Acetone and methanol are two important raw materials in the modern chemical industry. The acetone + methanol mixture presents a minimum azetrope at acetone mole fraction of $78 \%$, and the azeotropic temperature is $55.5^{\circ} \mathrm{C}$ at $101.3 \mathrm{kPa}$. Thus, it is difficult to separate this azeotropic system by common distillation. Several special distillation methods have been used to separate azeotropic mixtures, such as extractive distillation, salt adding distillation, and pressure swing distillation [1-5]. Because of the advantages of flexible selection of the entrainers, weak corrosiveness, low energy consumption and so on, extractive distillation has been widely applied to the separation of azeotropic or close-boiling mixtures. In the process of extractive distillation, it is essential to select a suitable entrainer with high stability, low toxicity, 
good solubility and high selectivity.

In recent years, ionic liquids (ILs) have been widely used as entrainers of extractive distillation since they showed the excellent abilities to separate azeotropic mixtures by Seiler et al [6-17]. ILs are composed of organic cations and inorganic or organic anions. ILs have many unique properties, such as negligible vapor pressure, good thermal stability, environmental friendliness. Arlt et al. [18] reported the isothermal vapor-liquid equilibrium (VLE) data for the ternary system of acetone + methanol + ILs (1-ethyl-3-methylimidazolium tetra-fluoroborate [EMIM] $\left[\mathrm{BF}_{4}\right]$ and N-butylpyri dinium hexafluoro-phosphate $\left.[\mathrm{BPY}]\left[\mathrm{PF}_{6}\right]\right)$ in 2002. Since then, several ionic liquids (e.g., 1-ethyl-3- methylimidazolium trifluoromethanesulfonate [emim][triflate], 1-butyl-3-ethylimidazolium trifluoromethanesulfonate [beim][triflate] 1-ethyl-3methylimidazolium dicyanamide [EMIM][DCA], 1-ethyl-3-methylimidazolium diethylphosphate [EMIM] [DEP], and 1-ethyl-3-methylimidazolium hydrogen sulfate, [EMIM] $\left.\left[\mathrm{HSO}_{4}\right][12,13,19-21]\right)$ have been investigated as solvents to separate this binary azeotropic system. The isothermal or the isobaric VLE data of these ternary systems containing ILs were obtained, and the azeotropic phenomenen for acetone + methanol system were totally broken at the mole fractions of ILs from 0.022 to 0.079. Li et al.[22,23] studied the influence of five ILs (monoethanolamine chloride [HMEA][Cl], monoethanolamine acetate [HMEA][OAC], 1-ethyl-3-methylimidazole acetate [EMIM][OAC], 1-ethyl-3-methylimidazolebromine [EMIM][Br] and 1-butyl-3-methylimidazole bromine [BMIM] $[\mathrm{Br}]$ ) on the VLE data of this binary system. The results showed that, the shorter the alkyl chain length on the imidazole ring of the IL cation is, the better the separation capacity.

In this work, the ILs investigated are three ILs with organic anions 1-methylimidazolium acetate [MIM]Ac, 1-methylimidazolium 2-hydroxypropionate [MIM]HPr, and 1-methylimidazolium propionate[MIM]Pr. The three ILs are without pollution and corrosion like the ILs containing inorganic anions such as $\mathrm{BF}_{4}^{-}, \mathrm{SO}_{4}{ }^{2-}$ and $\mathrm{Cl}^{-}$. They are also inexpensive and with short alkyl chain on the imidazole ring of the IL cations. The isobaric vapor-liquid equilibrium (VLE) data for the ternary systems of acetone + methanol containing different ILs were measured at $101.3 \mathrm{kPa}$, and the effects of the ILs on the separation performance of the acetone and methanol system were discussed.

\section{Experimental}

\subsection{Materials}

The chemicals used in this work were acetone (Sinopharm Group, minimum wt 99.5\%), methanol (Sinopharm Group, minimum wt 99.5\%), and ionic liquids (Lanzhou yulu fine chemical co. LTD, minimum wt 99.0\%). The organic solvents were checked by gas chromatograph. The water contents were measured by Karl Fischer titration. The three ILs were analyzed by liquid chromatography, and dried in 
a vacuum drying oven at $393 \mathrm{~K}$ overnight before experiment. Specifications of the chemical samples in this study are summarized in Table 1.

Table.1 Specifications of the chemical samples

\begin{tabular}{|c|c|c|c|c|c|c|}
\hline $\begin{array}{l}\text { Chemical } \\
\text { name }\end{array}$ & $\begin{array}{l}\text { CAS } \\
\text { RN }\end{array}$ & source & $\begin{array}{l}\text { Water } \\
\text { content }\end{array}$ & purity & $\begin{array}{c}\text { Purification } \\
\text { method }\end{array}$ & $\begin{array}{c}\text { Analysis } \\
\text { method }\end{array}$ \\
\hline acetone & $67-64-1$ & $\begin{array}{c}\text { Sinopharm } \\
\text { Group }\end{array}$ & none & 0.995 & none & $\mathrm{GC}^{\mathrm{a}}$ \\
\hline methanol & $67-56-1$ & $\begin{array}{l}\text { Sinopharm } \\
\text { Group }\end{array}$ & none & 0.995 & none & $\mathrm{GC}^{\mathrm{a}}$ \\
\hline$[\mathrm{MIM}] \mathrm{Ac}^{\mathrm{d}}$ & none & Yulu Group & $<5000 \mathrm{ppm}$ & 0.990 & $\begin{array}{c}\text { Vacuum } \\
\text { desiccation }\end{array}$ & $\mathrm{KF}^{\mathrm{b}} \mathrm{LC}^{\mathrm{c}}$ \\
\hline$[\mathrm{MIM}] \mathrm{Pr}^{\mathrm{e}}$ & none & Yulu Group & $<5000$ ppm & 0.990 & $\begin{array}{c}\text { Vacuum } \\
\text { desiccation }\end{array}$ & $\mathrm{KF}^{\mathrm{b}} \mathrm{LC}^{\mathrm{c}}$ \\
\hline$[\mathrm{MIM}] \mathrm{HPr}^{\mathrm{f}}$ & none & Yulu Group & $<5000$ ppm & 0.990 & $\begin{array}{c}\text { Vacuum } \\
\text { desiccation }\end{array}$ & $\mathrm{KF}^{\mathrm{b}} \mathrm{LC}^{\mathrm{c}}$ \\
\hline
\end{tabular}

${ }^{\mathrm{a}} \mathrm{GC}=$ gas chromatography. $\quad{ }^{\mathrm{b}} \mathrm{KF}=$ Karl Fischer titration. $\quad{ }^{\mathrm{c}} \mathrm{LC}=$ liquid chromatography.

${ }^{\mathrm{d}}[\mathrm{MIM}] \mathrm{Ac}=1$-methylimidazolium acetate $\quad{ }^{\mathrm{e}}[\mathrm{MIM}] \mathrm{Pr}=1$-methylimidazolium propionate

. $[\mathrm{MIM}] \mathrm{HPr}=1$-methylimidazolium 2-hydroxypropionate

\subsection{Apparatus and procedures}

All vapor-liquid equilibrium (VLE) experiments were conducted in an all-glass dynamic recirculating still (NGW, Wertheim, Germany) described by Hunsmann[24]. The temperature was detected by a calibrated thermometer with a standard uncertainty of $0.01 \mathrm{~K}$. The equilibrium pressure was kept constant at $101.3 \mathrm{kPa}$ via a gas buffer, and determined by a manometer with a standard uncertainty of $0.1 \mathrm{kPa}$. Each sample for the VLE experiment was gravimetrically prepared with an electronic balance (CAV264C OHAUS America), and the standard uncertainty is $0.1 \mathrm{mg}$.

The mole fractions of acetone and methanol were measured by using headspace sampler (G1888 headspace sampler, Agilent Technologies) and the gas chromatograph (model network 7890A, Agilent Technologies) described in the previous literatures $[22,23]$. After the temperature was stable for one hour, the condensed vapor and liquid phase samples taken from the dynamic recirculating still were inserted into the 
headspace sampler and tested by the gas chromatography (GC). The original reactants (1-methylimidazole, acetic acid, propionic acid and 2-hydroxyl propionic acid) were not found in the liquid and vapor phases by GC and liquid chromatography (LC). Due to its negligible vapor pressure, the IL content was gravimetrically determined by the mass difference after vaporizing the volatile components from a known mass of sample until constant mass. Each experiment was replicated at least three times, and the standard uncertainty of the mole composition is 0.001 .

\section{Results and discussion}

\subsection{Experimental data}

The VLE data for ternary systems of acetone (1) and methanol (2) containing [MIM]Ac (3), [MIM]HPr (3) and [MIM]Pr (3) were measured at 101.3kPa to examine the separation effects of ILs on acetone and methanol mixture. The mole fractions of ILs were kept nearly constant in series of $0.060,0.080$ and 0.100 in the liquid phase. The VLE data are listed in Tables 2-4, where T presents the equilibrium temperature, $\mathrm{x}_{\mathrm{i}}$ and $\mathrm{y}_{\mathrm{i}}$ are mole fractions of component $\mathrm{i}$ in the liquid and vapor phase, respectively. $\mathrm{x}_{1}$ ' presents the mole fraction of acetone in the liquid phase expressed on an IL-free basis, $\gamma_{i}$ is the activity coefficient of component $i, \alpha_{12}$ is the relative volatility of acetone to methanol.

Since the VLE data are measured at low total pressure of $101.3 \mathrm{KPa}$, the assumption of ideal vapor-phase can be made. The equation (1) is simply written as follows:

$$
\mathrm{y}_{\mathrm{i}} p=x_{\mathrm{i}} \gamma_{\mathrm{i}} p_{\mathrm{i}}^{\circ}
$$

Where $\mathrm{P}$ is the total pressure, and $\mathrm{P}_{\mathrm{i}}{ }^{0}$ is the saturated vapor pressure of component $\mathrm{i}$ at VLE temperature, which is calculated by Antoine equation (2). The Antoine equation is described as follows:

$$
\log P_{i}^{\circ}=A-\frac{B}{T+C}
$$

Where A, B and C are the Antoine parameters taken from previous literature [23]. The Antoine parameters are presented in Table 5. The relative volatility $\alpha_{12}$ is calculated by the following equation (3).

$$
\alpha_{12}=\frac{y_{1} / x_{1}}{y_{2} / x_{2}}=\frac{\gamma_{1}}{\gamma_{2}} \times \frac{\mathrm{p}_{1}^{\circ}}{\mathrm{p}_{2}^{\circ}}
$$


Table.2. Vapor-Liquid Equilibrium Data of the Ternary System Acetone (1) + Methanol (2) + [MIM]Ac (3) at $101.3 \mathrm{kPa}$

\begin{tabular}{|c|c|c|c|c|c|c|c|c|c|c|c|c|c|c|c|}
\hline $\mathrm{T} / \mathrm{K}$ & $\mathrm{X}_{3}$ & $\mathrm{X}_{2}$ & $\mathrm{x}_{1}$, & $\mathrm{y}_{1}$ & $\gamma_{1}$ & $\gamma_{2}$ & $\alpha_{12}$ & $\mathrm{~T} / \mathrm{K}$ & $\mathrm{X}_{3}$ & $\mathrm{X}_{2}$ & $\mathrm{x}_{1}$, & $\mathrm{y}_{1}$ & $\gamma_{1}$ & $\gamma_{2}$ & $\alpha_{12}$ \\
\hline 337.84 & 0.061 & 0.888 & 0.054 & 0.129 & 1.912 & 0.977 & 2.567 & 331.34 & 0.079 & 0.417 & 0.547 & 0.671 & 1.247 & 1.018 & 1.684 \\
\hline 336.34 & 0.059 & 0.841 & 0.106 & 0.226 & 1.797 & 0.973 & 2.451 & 331.16 & 0.081 & 0.364 & 0.604 & 0.708 & 1.203 & 1.042 & 1.591 \\
\hline 335.03 & 0.058 & 0.790 & 0.161 & 0.311 & 1.698 & 0.971 & 2.34 & 331.04 & 0.082 & 0.317 & 0.655 & 0.743 & 1.171 & 1.058 & 1.526 \\
\hline 334.07 & 0.062 & 0.741 & 0.210 & 0.374 & 1.629 & 0.977 & 2.248 & 330.93 & 0.083 & 0.271 & 0.704 & 0.773 & 1.134 & 1.096 & 1.426 \\
\hline 333.12 & 0.057 & 0.697 & 0.261 & 0.431 & 1.548 & 0.982 & 2.142 & 330.93 & 0.081 & 0.216 & 0.765 & 0.815 & 1.101 & 1.118 & 1.357 \\
\hline 332.49 & 0.061 & 0.644 & 0.314 & 0.482 & 1.474 & 0.992 & 2.027 & 331.01 & 0.080 & 0.169 & 0.816 & 0.851 & 1.071 & 1.148 & 1.285 \\
\hline 331.77 & 0.062 & 0.585 & 0.376 & 0.536 & 1.405 & 1.006 & 1.914 & 331.21 & 0.079 & 0.115 & 0.875 & 0.894 & 1.042 & 1.195 & 1.200 \\
\hline 331.39 & 0.060 & 0.539 & 0.427 & 0.574 & 1.339 & 1.018 & 1.807 & 331.72 & 0.081 & 0.022 & 0.976 & 0.977 & 1.005 & 1.328 & 1.037 \\
\hline 331.02 & 0.061 & 0.484 & 0.485 & 0.616 & 1.283 & 1.036 & 1.707 & 339.58 & 0.098 & 0.851 & 0.057 & 0.141 & 1.973 & 0.935 & 2.734 \\
\hline 330.73 & 0.062 & 0.434 & 0.537 & 0.651 & 1.237 & 1.063 & 1.608 & 337.77 & 0.101 & 0.799 & 0.111 & 0.247 & 1.872 & 0.936 & 2.625 \\
\hline 330.50 & 0.059 & 0.386 & 0.590 & 0.686 & 1.190 & 1.083 & 1.519 & 336.26 & 0.102 & 0.746 & 0.169 & 0.338 & 1.764 & 0.936 & 2.500 \\
\hline 330.31 & 0.060 & 0.339 & 0.639 & 0.720 & 1.159 & 1.110 & 1.447 & 335.12 & 0.097 & 0.706 & 0.218 & 0.405 & 1.693 & 0.929 & 2.436 \\
\hline 330.25 & 0.062 & 0.292 & 0.689 & 0.754 & 1.132 & 1.136 & 1.382 & 334.16 & 0.102 & 0.652 & 0.274 & 0.464 & 1.605 & 0.942 & 2.294 \\
\hline 330.19 & 0.068 & 0.229 & 0.754 & 0.792 & 1.096 & 1.223 & 1.243 & 333.45 & 0.101 & 0.604 & 0.328 & 0.517 & 1.527 & 0.942 & 2.193 \\
\hline 330.22 & 0.061 & 0.188 & 0.800 & 0.831 & 1.075 & 1.210 & 1.231 & 332.83 & 0.099 & 0.548 & 0.392 & 0.571 & 1.440 & 0.946 & 2.070 \\
\hline 330.41 & 0.062 & 0.132 & 0.859 & 0.873 & 1.046 & 1.286 & 1.126 & 332.49 & 0.098 & 0.501 & 0.445 & 0.612 & 1.376 & 0.954 & 1.967 \\
\hline 330.94 & 0.059 & 0.044 & 0.953 & 0.955 & 1.010 & 1.328 & 1.048 & 332.08 & 0.101 & 0.444 & 0.506 & 0.655 & 1.317 & 0.972 & 1.853 \\
\hline 338.71 & 0.082 & 0.867 & 0.056 & 0.134 & 1.941 & 0.960 & 2.637 & 331.80 & 0.099 & 0.397 & 0.559 & 0.691 & 1.266 & 0.985 & 1.761 \\
\hline 337.14 & 0.078 & 0.822 & 0.108 & 0.236 & 1.831 & 0.950 & 2.542 & 331.59 & 0.102 & 0.343 & 0.618 & 0.729 & 1.220 & 1.007 & 1.662 \\
\hline 335.69 & 0.081 & 0.767 & 0.165 & 0.325 & 1.738 & 0.953 & 2.431 & 331.54 & 0.099 & 0.300 & 0.667 & 0.760 & 1.178 & 1.021 & 1.584 \\
\hline 334.73 & 0.080 & 0.723 & 0.214 & 0.387 & 1.650 & 0.954 & 2.321 & 331.51 & 0.102 & 0.252 & 0.719 & 0.794 & 1.144 & 1.046 & 1.501 \\
\hline 333.79 & 0.079 & 0.675 & 0.267 & 0.446 & 1.569 & 0.958 & 2.213 & 331.53 & 0.101 & 0.196 & 0.782 & 0.836 & 1.105 & 1.066 & 1.423 \\
\hline 333.11 & 0.082 & 0.623 & 0.321 & 0.499 & 1.500 & 0.968 & 2.103 & 331.71 & 0.104 & 0.145 & 0.838 & 0.872 & 1.072 & 1.120 & 1.312 \\
\hline 332.46 & 0.081 & 0.566 & 0.384 & 0.554 & 1.420 & 0.974 & 1.990 & 332.07 & 0.099 & 0.095 & 0.895 & 0.915 & 1.035 & 1.121 & 1.262 \\
\hline 331.99 & 0.082 & 0.517 & 0.437 & 0.596 & 1.365 & 0.985 & 1.898 & 332.74 & 0.102 & 0.001 & 0.999 & 0.999 & 0.994 & 1.214 & 1.114 \\
\hline 331.58 & 0.083 & 0.462 & 0.496 & 0.635 & 1.300 & 1.009 & 1.769 & & & & & & & & \\
\hline
\end{tabular}

Standard uncertainty $\mathrm{u}\left(\mathrm{x}_{1}\right)=\mathrm{u}\left(\mathrm{y}_{1}\right)=0.001, \mathrm{u}(\mathrm{T})=0.01 \mathrm{~K}$ 
Table.3. Vapor-Liquid Equilibrium Data of the Ternary System Acetone (1) + Methanol (2) + [MIM] HPr (3) at 101.3kPa

\begin{tabular}{|c|c|c|c|c|c|c|c|c|c|c|c|c|c|c|c|}
\hline $\mathrm{T} / \mathrm{K}$ & $\mathrm{X}_{3}$ & $\mathrm{X}_{2}$ & $x_{1}{ }^{\prime}$ & $\mathrm{y}_{1}$ & $\gamma_{1}$ & $\gamma_{2}$ & $\alpha_{12}$ & $\mathrm{~T} / \mathrm{K}$ & $\mathrm{X}_{3}$ & $\mathrm{X}_{2}$ & $\mathrm{x}_{1}{ }^{\prime}$ & $\mathrm{y}_{1}$ & $\gamma_{1}$ & $\gamma_{2}$ & $\alpha_{12}$ \\
\hline 337.79 & 0.061 & 0.888 & 0.054 & 0.131 & 1.942 & 0.976 & 2.613 & 331.25 & 0.079 & 0.417 & 0.547 & 0.670 & 1.251 & 1.023 & 1.681 \\
\hline 336.28 & 0.059 & 0.841 & 0.106 & 0.228 & 1.813 & 0.971 & 2.477 & 331.09 & 0.081 & 0.364 & 0.604 & 0.707 & 1.204 & 1.049 & 1.582 \\
\hline 334.89 & 0.058 & 0.790 & 0.161 & 0.313 & 1.720 & 0.974 & 2.368 & 330.91 & 0.082 & 0.317 & 0.655 & 0.740 & 1.169 & 1.074 & 1.500 \\
\hline 333.93 & 0.062 & 0.741 & 0.210 & 0.372 & 1.625 & 0.985 & 2.226 & 330.90 & 0.080 & 0.274 & 0.702 & 0.771 & 1.134 & 1.096 & 1.427 \\
\hline 333.13 & 0.059 & 0.695 & 0.261 & 0.432 & 1.554 & 0.981 & 2.150 & 330.85 & 0.081 & 0.216 & 0.765 & 0.813 & 1.100 & 1.134 & 1.338 \\
\hline 332.36 & 0.061 & 0.644 & 0.314 & 0.483 & 1.488 & 0.994 & 2.043 & 330.93 & 0.080 & 0.169 & 0.816 & 0.849 & 1.072 & 1.173 & 1.260 \\
\hline 331.72 & 0.062 & 0.585 & 0.376 & 0.537 & 1.411 & 1.006 & 1.923 & 331.10 & 0.079 & 0.115 & 0.875 & 0.892 & 1.041 & 1.220 & 1.175 \\
\hline 331.39 & 0.057 & 0.542 & 0.425 & 0.574 & 1.343 & 1.013 & 1.823 & 331.70 & 0.081 & 0.022 & 0.976 & 0.976 & 1.006 & 1.372 & 1.006 \\
\hline 330.89 & 0.061 & 0.484 & 0.485 & 0.617 & 1.290 & 1.041 & 1.710 & 339.39 & 0.098 & 0.851 & 0.057 & 0.144 & 1.909 & 0.942 & 2.800 \\
\hline 330.63 & 0.058 & 0.438 & 0.535 & 0.649 & 1.237 & 1.064 & 1.608 & 337.78 & 0.101 & 0.799 & 0.111 & 0.251 & 1.793 & 0.935 & 2.682 \\
\hline 330.48 & 0.059 & 0.386 & 0.590 & 0.685 & 1.191 & 1.089 & 1.514 & 336.27 & 0.103 & 0.745 & 0.169 & 0.342 & 1.720 & 0.936 & 2.544 \\
\hline 330.34 & 0.061 & 0.338 & 0.640 & 0.718 & 1.158 & 1.119 & 1.434 & 335.15 & 0.102 & 0.701 & 0.219 & 0.409 & 1.621 & 0.935 & 2.461 \\
\hline 330.21 & 0.062 & 0.292 & 0.689 & 0.752 & 1.131 & 1.144 & 1.371 & 334.22 & 0.097 & 0.657 & 0.272 & 0.467 & 1.541 & 0.933 & 2.341 \\
\hline 330.20 & 0.063 & 0.234 & 0.750 & 0.791 & 1.094 & 1.206 & 1.257 & 333.53 & 0.101 & 0.604 & 0.328 & 0.521 & 1.451 & 0.937 & 2.226 \\
\hline 330.28 & 0.061 & 0.188 & 0.800 & 0.828 & 1.070 & 1.231 & 1.205 & 332.86 & 0.102 & 0.545 & 0.393 & 0.574 & 1.384 & 0.950 & 2.079 \\
\hline 330.47 & 0.062 & 0.132 & 0.859 & 0.871 & 1.041 & 1.303 & 1.106 & 332.39 & 0.099 & 0.500 & 0.445 & 0.612 & 1.318 & 0.959 & 1.969 \\
\hline 331.02 & 0.061 & 0.042 & 0.955 & 0.954 & 1.006 & 1.417 & 0.978 & 332.05 & 0.101 & 0.444 & 0.506 & 0.655 & 1.257 & 0.973 & 1.853 \\
\hline 338.67 & 0.078 & 0.871 & 0.055 & 0.136 & 1.975 & 0.955 & 2.697 & 331.84 & 0.099 & 0.397 & 0.559 & 0.687 & 1.217 & 0.995 & 1.731 \\
\hline 336.89 & 0.080 & 0.820 & 0.109 & 0.240 & 1.874 & 0.959 & 2.582 & 331.64 & 0.101 & 0.344 & 0.617 & 0.728 & 1.182 & 1.009 & 1.655 \\
\hline 335.53 & 0.081 & 0.767 & 0.165 & 0.328 & 1.764 & 0.954 & 2.465 & 331.47 & 0.099 & 0.300 & 0.667 & 0.762 & 1.143 & 1.016 & 1.597 \\
\hline 334.48 & 0.083 & 0.72 & 0.215 & 0.391 & 1.677 & 0.962 & 2.345 & 331.42 & 0.102 & 0.252 & 0.719 & 0.791 & 1.107 & 1.061 & 1.480 \\
\hline 333.73 & 0.079 & 0.675 & 0.267 & 0.451 & 1.588 & 0.953 & 2.251 & 331.48 & 0.101 & 0.196 & 0.782 & 0.834 & 1.078 & 1.085 & 1.401 \\
\hline 333.00 & 0.082 & 0.623 & 0.321 & 0.501 & 1.508 & 0.967 & 2.119 & 331.52 & 0.100 & 0.149 & 0.834 & 0.870 & 1.049 & 1.112 & 1.331 \\
\hline 332.39 & 0.081 & 0.566 & 0.384 & 0.555 & 1.425 & 0.974 & 1.997 & 331.61 & 0.099 & 0.095 & 0.895 & 0.913 & 1.021 & 1.171 & 1.229 \\
\hline 331.93 & 0.082 & 0.517 & 0.437 & 0.596 & 1.369 & 0.985 & 1.904 & 331.90 & 0.102 & 0.001 & 0.999 & 0.999 & 1.015 & 1.255 & 1.114 \\
\hline 331.49 & 0.078 & 0.467 & 0.493 & 0.636 & 1.303 & 1.000 & 1.791 & & & & & & & & \\
\hline
\end{tabular}

Standard uncertainty $\mathrm{u}\left(\mathrm{x}_{1}\right)=\mathrm{u}\left(\mathrm{y}_{1}\right)=0.001, \mathrm{u}(\mathrm{T})=0.01 \mathrm{~K}$ 
Table.4. Vapor-Liquid Equilibrium Data of the Ternary System Acetone (1) + Methanol (2) + [MIM]Pr (3) at 101.3kPa

\begin{tabular}{|c|c|c|c|c|c|c|c|c|c|c|c|c|c|c|c|}
\hline $\mathrm{T} / \mathrm{K}$ & $\mathrm{x}_{3}$ & $\mathrm{x}_{2}$ & $\mathrm{x}_{1}{ }^{\prime}$ & $\mathrm{y}_{1}$ & $\gamma_{1}$ & $\gamma_{2}$ & $\alpha_{12}$ & $\mathrm{~T} / \mathrm{K}$ & $\mathrm{x}_{3}$ & $\mathrm{X}_{2}$ & $x_{1}{ }^{\prime}$ & $\mathrm{y}_{1}$ & $\gamma_{1}$ & $\gamma_{2}$ & $\alpha_{12}$ \\
\hline 337.69 & 0.061 & 0.888 & 0.054 & 0.126 & 1.885 & 0.986 & 2.512 & 331.24 & 0.079 & 0.416 & 0.548 & 0.659 & 1.228 & 1.061 & 1.592 \\
\hline 336.15 & 0.059 & 0.838 & 0.109 & 0.227 & 1.764 & 0.984 & 2.382 & 331.04 & 0.078 & 0.367 & 0.602 & 0.696 & 1.188 & 1.081 & 1.515 \\
\hline 334.79 & 0.061 & 0.787 & 0.162 & 0.305 & 1.680 & 0.991 & 2.274 & 330.97 & 0.082 & 0.316 & 0.656 & 0.732 & 1.154 & 1.111 & 1.432 \\
\hline 333.89 & 0.062 & 0.741 & 0.210 & 0.367 & 1.606 & 0.994 & 2.182 & 330.84 & 0.084 & 0.268 & 0.707 & 0.763 & 1.121 & 1.161 & 1.331 \\
\hline 333.12 & 0.063 & 0.691 & 0.263 & 0.423 & 1.521 & 1.005 & 2.055 & 330.92 & 0.081 & 0.216 & 0.765 & 0.805 & 1.086 & 1.183 & 1.267 \\
\hline 332.39 & 0.061 & 0.644 & 0.314 & 0.473 & 1.454 & 1.012 & 1.961 & 331.04 & 0.082 & 0.166 & 0.819 & 0.842 & 1.058 & 1.237 & 1.178 \\
\hline 331.73 & 0.062 & 0.585 & 0.376 & 0.527 & 1.383 & 1.028 & 1.844 & 331.31 & 0.079 & 0.115 & 0.875 & 0.888 & 1.032 & 1.254 & 1.131 \\
\hline 331.32 & 0.058 & 0.540 & 0.427 & 0.565 & 1.318 & 1.040 & 1.743 & 332.02 & 0.080 & 0.023 & 0.975 & 0.975 & 0.994 & 1.364 & 0.996 \\
\hline 330.90 & 0.061 & 0.483 & 0.486 & 0.607 & 1.267 & 1.068 & 1.636 & 339.00 & 0.098 & 0.851 & 0.057 & 0.137 & 1.968 & 0.964 & 2.658 \\
\hline 330.66 & 0.057 & 0.438 & 0.536 & 0.642 & 1.219 & 1.085 & 1.553 & 337.39 & 0.101 & 0.796 & 0.115 & 0.247 & 1.846 & 0.958 & 2.536 \\
\hline 330.38 & 0.059 & 0.386 & 0.590 & 0.676 & 1.177 & 1.123 & 1.452 & 336.01 & 0.103 & 0.745 & 0.169 & 0.331 & 1.752 & 0.961 & 2.424 \\
\hline 330.19 & 0.057 & 0.341 & 0.638 & 0.710 & 1.147 & 1.147 & 1.386 & 334.93 & 0.102 & 0.701 & 0.219 & 0.395 & 1.674 & 0.964 & 2.327 \\
\hline 330.09 & 0.062 & 0.290 & 0.691 & 0.745 & 1.122 & 1.191 & 1.307 & 334.13 & 0.097 & 0.657 & 0.272 & 0.453 & 1.578 & 0.961 & 2.214 \\
\hline 330.01 & 0.059 & 0.238 & 0.747 & 0.783 & 1.090 & 1.239 & 1.221 & 333.43 & 0.101 & 0.604 & 0.328 & 0.506 & 1.503 & 0.973 & 2.093 \\
\hline 330.04 & 0.061 & 0.187 & 0.801 & 0.822 & 1.069 & 1.292 & 1.148 & 332.73 & 0.103 & 0.544 & 0.394 & 0.559 & 1.419 & 0.991 & 1.951 \\
\hline 330.24 & 0.056 & 0.138 & 0.854 & 0.866 & 1.043 & 1.309 & 1.105 & 332.38 & 0.099 & 0.499 & 0.446 & 0.599 & 1.352 & 0.995 & 1.855 \\
\hline 331.01 & 0.064 & 0.039 & 0.958 & 0.952 & 1.004 & 1.593 & 0.868 & 331.99 & 0.104 & 0.440 & 0.509 & 0.643 & 1.295 & 1.022 & 1.735 \\
\hline 338.25 & 0.077 & 0.872 & 0.055 & 0.131 & 1.925 & 0.976 & 2.580 & 331.62 & 0.099 & 0.396 & 0.560 & 0.678 & 1.247 & 1.036 & 1.651 \\
\hline 336.59 & 0.080 & 0.817 & 0.112 & 0.237 & 1.813 & 0.976 & 2.460 & 331.45 & 0.096 & 0.349 & 0.614 & 0.715 & 1.204 & 1.047 & 1.579 \\
\hline 335.43 & 0.081 & 0.767 & 0.165 & 0.318 & 1.715 & 0.973 & 2.354 & 331.32 & 0.099 & 0.299 & 0.668 & 0.748 & 1.166 & 1.088 & 1.473 \\
\hline 334.49 & 0.078 & 0.725 & 0.214 & 0.380 & 1.630 & 0.974 & 2.251 & 331.23 & 0.095 & 0.257 & 0.716 & 0.783 & 1.135 & 1.090 & 1.433 \\
\hline 333.58 & 0.079 & 0.675 & 0.267 & 0.436 & 1.546 & 0.985 & 2.125 & 331.25 & 0.101 & 0.196 & 0.782 & 0.825 & 1.101 & 1.151 & 1.315 \\
\hline 332.95 & 0.083 & 0.622 & 0.322 & 0.488 & 1.473 & 0.996 & 2.010 & 331.31 & 0.100 & 0.148 & 0.836 & 0.863 & 1.074 & 1.189 & 1.242 \\
\hline 332.28 & 0.081 & 0.566 & 0.384 & 0.543 & 1.399 & 1.003 & 1.905 & 331.52 & 0.099 & 0.095 & 0.895 & 0.908 & 1.047 & 1.239 & 1.161 \\
\hline 331.87 & 0.082 & 0.516 & 0.438 & 0.584 & 1.340 & 1.019 & 1.802 & 332.06 & 0.102 & 0.001 & 0.999 & 0.999 & 1.016 & 1.397 & 0.994 \\
\hline 331.48 & 0.083 & 0.461 & 0.497 & 0.624 & 1.280 & 1.048 & 1.678 & & & & & & & & \\
\hline
\end{tabular}

Standard uncertainty $\mathrm{u}\left(\mathrm{x}_{1}\right)=\mathrm{u}\left(\mathrm{y}_{1}\right)=0.001, \mathrm{u}(\mathrm{T})=0.01 \mathrm{~K}$

Table.5. Antoine's Parameter for Pure Components

\begin{tabular}{cccc}
\hline component & A & B & C \\
\hline acetone & 6.3565 & 1277.0 & -35.920 \\
methanol & 7.0224 & 1474.1 & -44.020 \\
Parameter obtained from ref 23 & & & \\
\hline
\end{tabular}


Table.6. Binary Energy Parameters and Average Relative Deviations

\begin{tabular}{cccccc}
\hline component $i$ & component $i$ & $\alpha_{\mathrm{i}, \mathrm{j}}$ & $\Delta \mathrm{g}_{\mathrm{i}, \mathrm{j}} /(\mathrm{J} / \mathrm{mol})$ & $\Delta \mathrm{g}_{\mathrm{j}, \mathrm{i}} /(\mathrm{J} / \mathrm{mol})$ & ARD \\
\hline acetone & methanol & 0.300 & 924.200 & 863.10 & $1.6 \%$ \\
acetone & {$[\mathrm{MIM}] \mathrm{Ac}$} & 0.100 & -3408.740 & 1829.08 & $0.6 \%$ \\
methanol & {$[\mathrm{MIM}] \mathrm{Ac}$} & 0.680 & -3533.450 & -3325.60 & \\
acetone & {$[\mathrm{MIM}] \mathrm{HPr}$} & 0.100 & -3491.880 & 3325.60 & $0.2 \%$ \\
methanol & {$[\mathrm{MIM}] \mathrm{HPr}$} & 0.520 & -2494.200 & -3658.16 & \\
acetone & {$[\mathrm{MIM}] \operatorname{Pr}$} & 0.220 & -6958.818 & 4157.00 & $0.7 \%$ \\
methanol & {$[\mathrm{MIM}] \mathrm{Pr}$} & 0.690 & -3325.600 & -4073.86 & \\
\hline
\end{tabular}

$\Delta g_{i, j} g_{i, j}-g_{j, i}, \tau_{i j}=\left(g_{i, j}-g_{j, i}\right) / R T$

\subsection{Correlation of the phase equilibrium}

In this work, the VLE data have been correlated by using the nonrandom two-liquid (NRTL) model, and regressed through minimizing the average relative deviations (ARD) function in the following equation (4) by levenberg-Marquardt method. There are six binary energy parameters and three nonrandom factors in the model. The nine parameters are obtained from the ternary VLE data in tables 2-4.

$$
A R D(\%)=\frac{1}{n} \sum_{n} \sum_{i}\left|\frac{y_{i}^{\exp t l}-y_{i}^{\text {calcd }}}{y_{i}^{\exp t l}}\right| \times 100
$$

Where $\mathrm{n}$ denotes the number of experiment points, $y_{\mathrm{i}}$ is mole fraction of solvent $i$ in the vapor phase, and the superscripts "exptl" and "calcd" represent experimental and calculated values, respectively. The binary interaction parameters and the average relative deviations (ARD) are shown in Table 6, and the experimental values and the correlated diagrams are drawn in Figs 1-15. As can be seen, the experimental values are quite well correlated by the NRTL model.

Figs 1-6 show the $\mathrm{y}_{1}-\mathrm{x}_{1}$ ' diagrams and the $\mathrm{T}-\mathrm{x}_{1}$ '- $\mathrm{y}_{1}$ diagrams for acetone $(1)+$ methanol (2) + [MIM]Ac (3), [MIM]HPr (3) or [MIM]Pr (3) ternary systems on an IL-free basis, respectively. It can be seen that the addition of an IL makes the mole fraction of acetone in the vapor phase increased, and this effect is more notable with the increase of the content of the IL. The addition of an IL shifts upward the azeotropic point of acetone and methanol, and the azeotropy can be totally eliminated by increasing the mole fraction of the IL to a specific value. According to the NRTL model, the minimum mole contents of [MIM]Ac, [MIM]HPr and [MIM]Pr needed to eliminate the azeotropy are 0.067, 0.074 and 0.089, respectively. Therefore, [MIM]Ac has more remarkable azeotropy breaking capacity for acetone + methanol system than [MIM]HPr and [MIM]Pr. The VLE temperature also increases with the addition of an IL. 


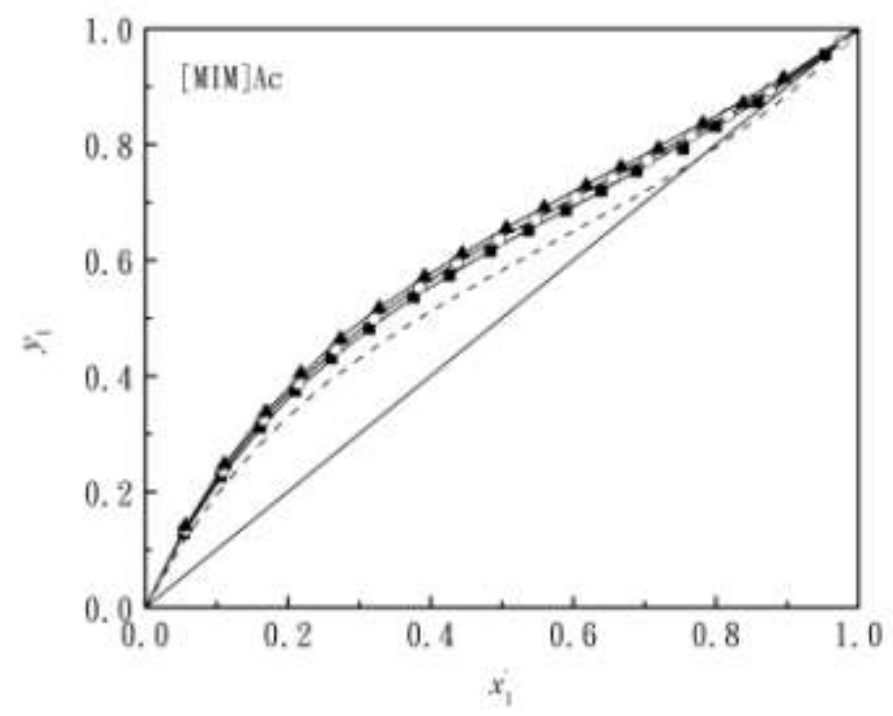

Fig.1. $y-x^{9}$ diagrams for acetone (1) + methanol (2) + [MIM]Ac (3) system in $x-y$ diagram at $101.3 \mathrm{kPa}$; dotted line, IL-free system; $\mathrm{n}, \mathrm{x}_{3}=0.06 ; \circ, \mathrm{x}_{3}=0.08 ; \Delta, \mathrm{x}_{3}=0.1$; solid line, correlated using NRTL model.

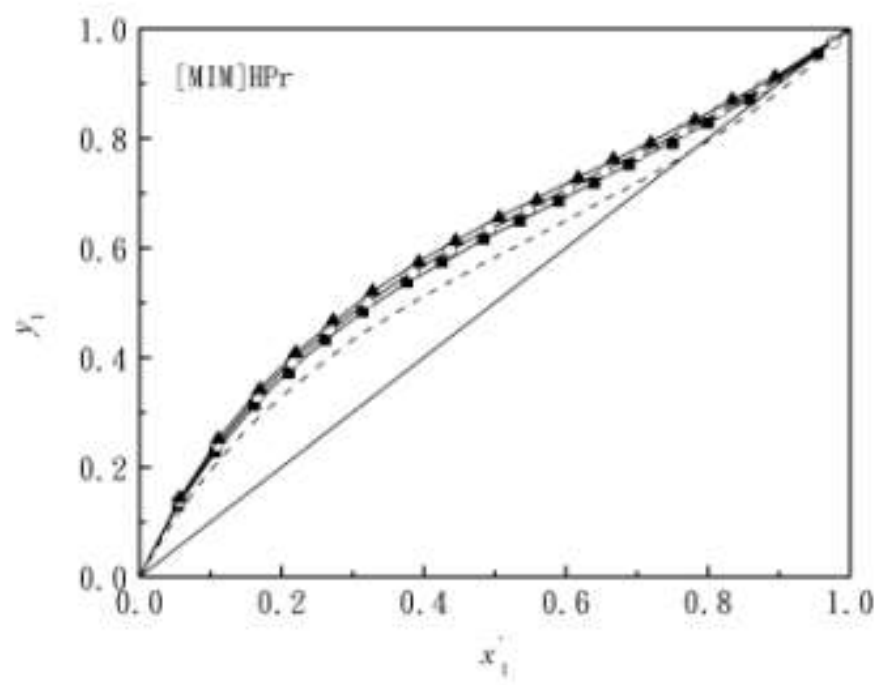

Fig.2. $y-x^{9}$ diagrams for acetone (1) + methanol (2) + [MIM]HPr (3) system in $x-y$ diagram at $101.3 \mathrm{kPa}$; dotted line, IL- free system; $n, x_{3}=0.06 ; \circ, x_{3}=0.08 ; \Delta, x_{3}=0.1$; solid line, correlated using NRTL model. 


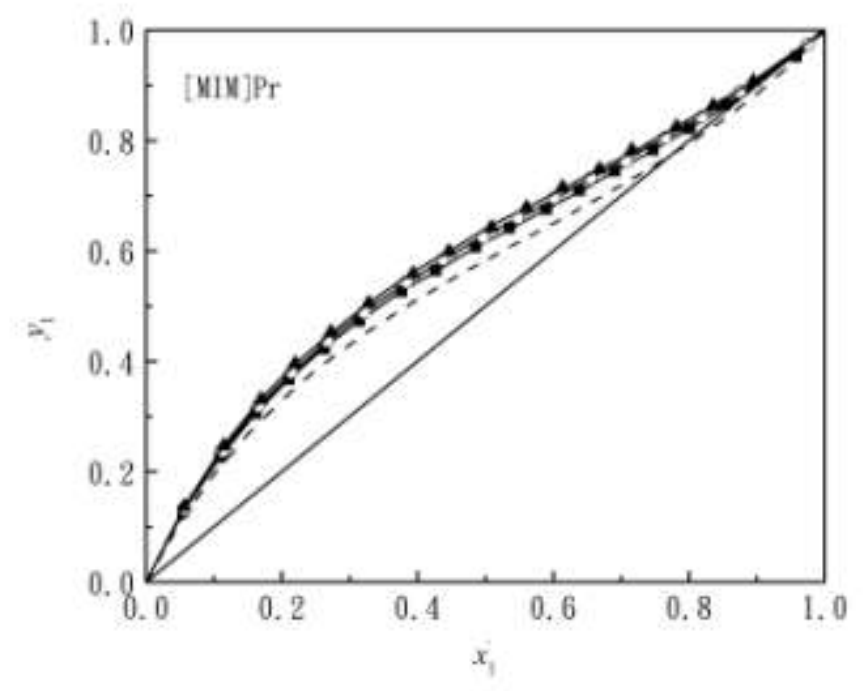

Fig.3. $y-x^{\prime}$ diagrams for acetone (1) + methanol (2) + [MIM] $\operatorname{Pr}(3)$ system in $x-y$ diagram at $101.3 \mathrm{kPa}$; dotted line, IL-free system; $m, \mathrm{x}_{3}=0.06 ; \circ, \mathrm{x}_{3}=0.08 ; \Delta, \mathrm{x}_{3}=0.1$; solid line, correlated using NRTL model.

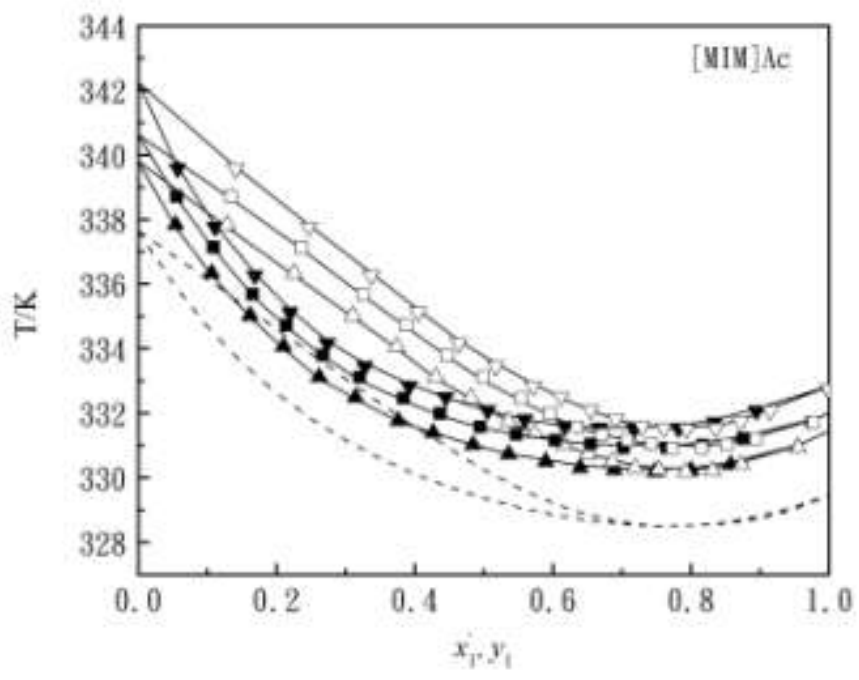

Fig.4. T- $x^{\prime}{ }_{1}-y_{1}$ diagrams for the ternary system of acetone (1) + methanol (2) + [MIM]Ac (3) at $101.3 \mathrm{kPa}$ : dotted line, IL-free system; $\Delta, \mathrm{x}^{\prime}{ }_{1}\left(\mathrm{x}_{3}=0.06\right) ; \triangle, \mathrm{y}_{1}\left(\mathrm{x}_{3}=0.06\right) ; \square, \mathrm{x}_{1}{ }_{1}\left(\mathrm{x}_{3}=\right.$ $0.08) ; \square, y_{1}\left(x_{3}=0.08\right) ; \nabla, x^{\prime}{ }_{1}\left(x_{3}=0.1\right) ; \nabla, y_{1}\left(x_{3}=0.1\right)$; solid line, correlated using NRTL model. 


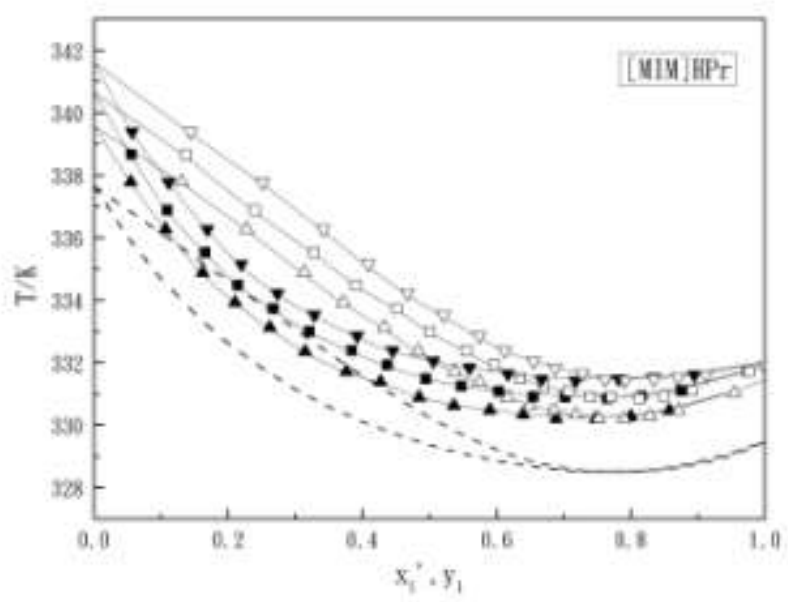

Fig.5. T-x' ${ }^{\prime}-y_{1}$ diagrams for the ternary system of acetone (1) + methanol (2) + [MIM]HPr (3) at $101.3 \mathrm{kPa}$ : dotted line, IL-free system; $\Delta, \mathrm{x}^{\prime}{ }_{1}\left(\mathrm{x}_{3}=0.06\right) ; \triangle, \mathrm{y}_{1}\left(\mathrm{x}_{3}=0.06\right) ; \mathrm{n}, \mathrm{x}_{1}^{\prime}{ }_{1}\left(\mathrm{x}_{3}=\right.$ $0.08) ; \square, y_{1}\left(x_{3}=0.08\right) ; \nabla, x_{1}^{\prime}\left(x_{3}=0.1\right) ; \nabla, y_{1}\left(x_{3}=0.1\right)$; solid line, correlated using NRTL model.

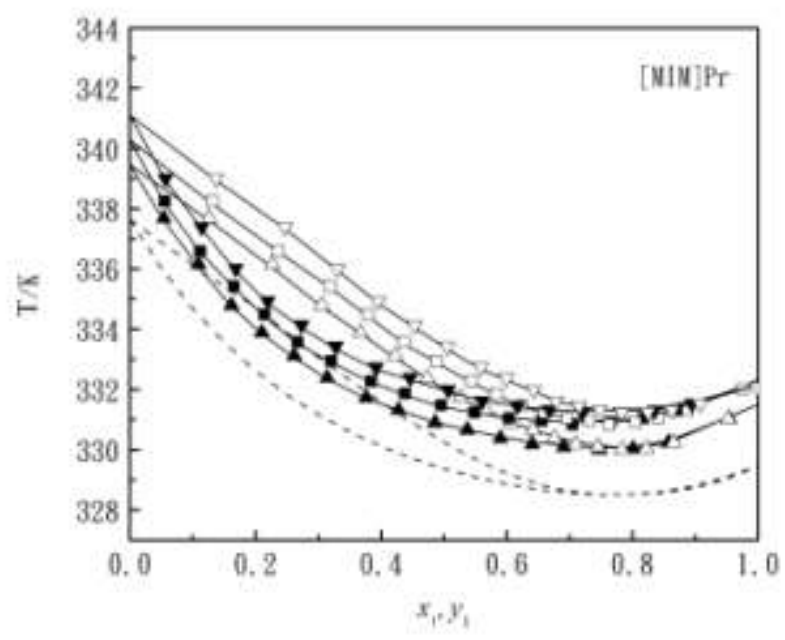

Fig.6. $T-x^{\prime}{ }_{1}-y_{1}$ diagrams for the ternary system of acetone (1) + methanol (2) + [MIM] $\operatorname{Pr}(3)$ at $101.3 \mathrm{kPa}$ : dotted line, IL-free system; $\Delta, \mathrm{x}^{\prime}{ }_{1}\left(\mathrm{x}_{3}=0.06\right) ; \triangle, \mathrm{y}_{1}\left(\mathrm{x}_{3}=0.06\right) ; n, \mathrm{x}^{\prime}{ }_{1}\left(\mathrm{x}_{3}=\right.$ $0.08) ; \square, \mathrm{y}_{1}\left(\mathrm{x}_{3}=0.08\right) ; \nabla, \mathrm{x}^{\prime}{ }_{1}\left(\mathrm{x}_{3}=0.1\right) ; \nabla, \mathrm{y}_{1}\left(\mathrm{x}_{3}=0.1\right)$; solid line, correlated using NRTL model. 


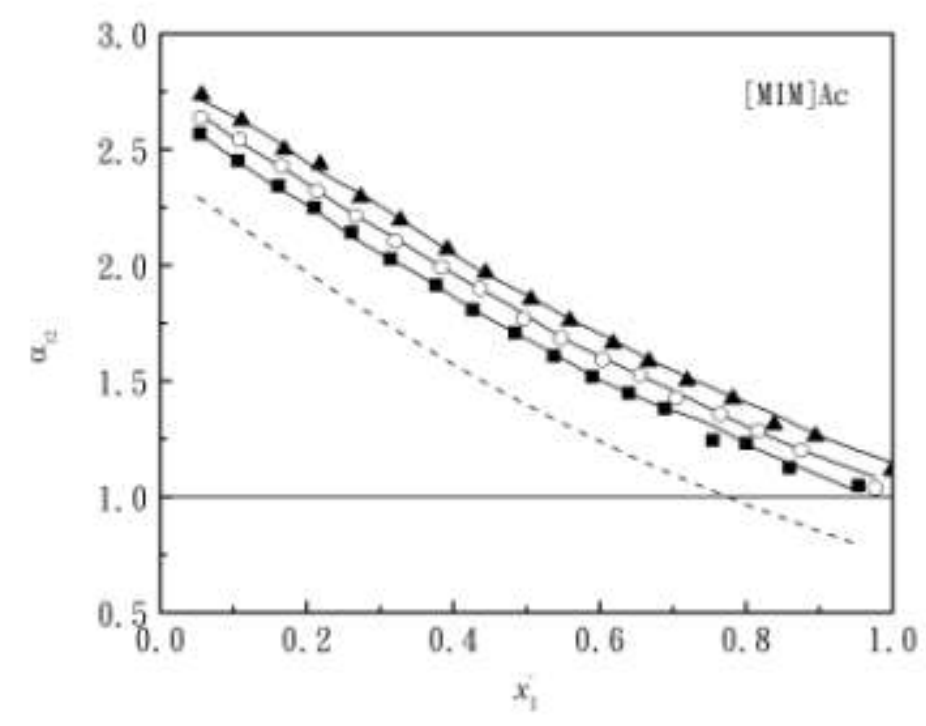

Fig.7. Separation factor of acetone (1) to methanol (2) at different mole fraction of [MIM]Ac at 101.3kPa: dotted line, IL-free system; $n, x_{3}=0.06 ; \circ, x_{3}=0.08 ; \Delta, x_{3}=0.1$; solid line, correlated using the NRTL model.

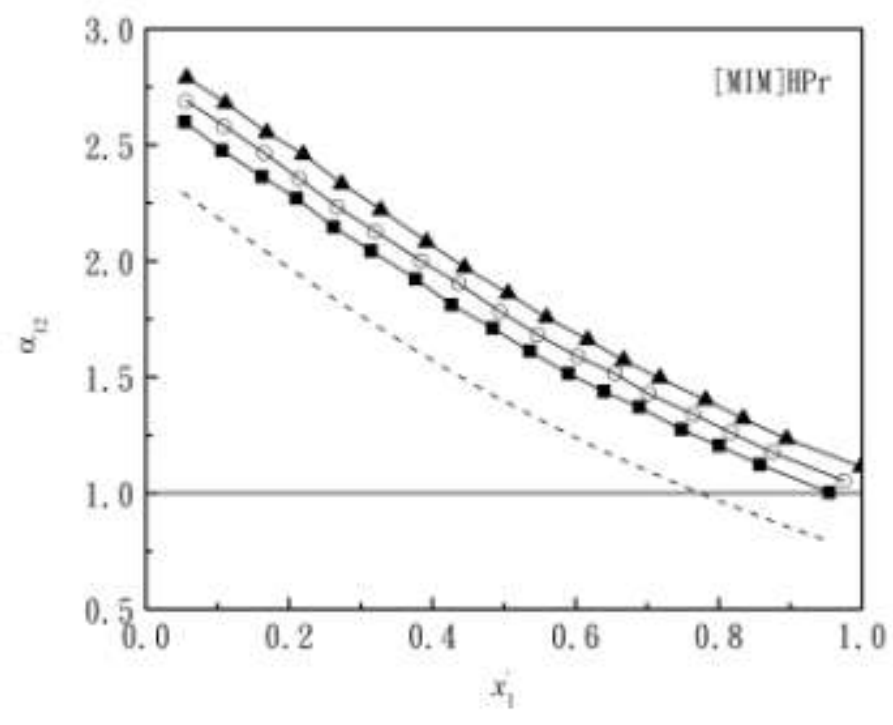

Fig.8.Separation factor of acetone (1) to methanol (2) at different mole fraction of [MIM] HPr at 101.3kPa: dotted line, IL-free system; $n, x_{3}=0.06 ; \circ, x_{3}=0.08 ; \Delta, x_{3}=0.1$; solid line, correlated using the NRTL model. 


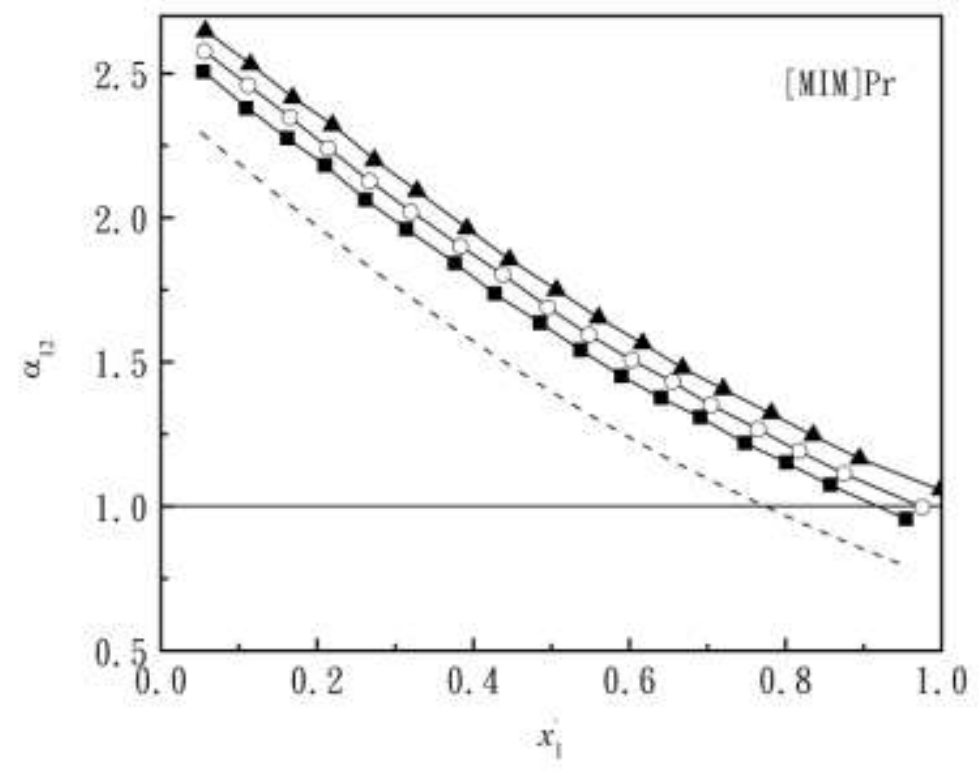

Fig.9. Separation factor of acetone (1) to methanol (2) at different mole fraction of [MIM] Pr at 101.3kPa: dotted line, IL- free system; $\mathrm{m}, \mathrm{x}_{3}=0.06 ; \circ, \mathrm{x}_{3}=0.08 ; \Delta, \mathrm{x}_{3}=0.1$; solid line, correlated using the NRTL model.

As the separation ability of an entrainer can be expressed by the relative volatility, the relative volatilities of acetone to methanol for the ternary systems containing ILs ([MIM]Ac, [MIM]HPr or [MIM]Pr) are calculated and drawn in Figs 7-9. The relative volatility significantly increases with the addition of an IL. The more ILs are, the larger the relative volatility, consequently the easier the separation. The values of relative volatility are higher than 1 on the whole studied concentration range when the mole fractions of the three ILs are 0.1. The above results are consistent with the previous conclusions. It is worth noting that $\alpha_{12}$ produced by [MIM]HPr is obviously greater than that produced by [MIM]Ac at areas of higher methanol content. The complex effects of the two ILs on the mixture of acetone and methanol will be discussed in the later chapters.

The activity coefficients of acetone $\left(\gamma_{1}\right)$ and methanol $\left(\gamma_{2}\right)$ in the ternary systems with different mole fractions of ILs are plotted in Figs 10-15 to verify the effects of ILs on the solution non-ideality. It can be seen that $\gamma_{1}$ increase while $\gamma_{2}$ decrease with the increase of IL. This kind of change illustrates that ILs have repulsive forces to acetone and attractive forces to methanol, pushing acetone to the vapor phase, thus enhancing the relative volatility of acetone to methanol. 


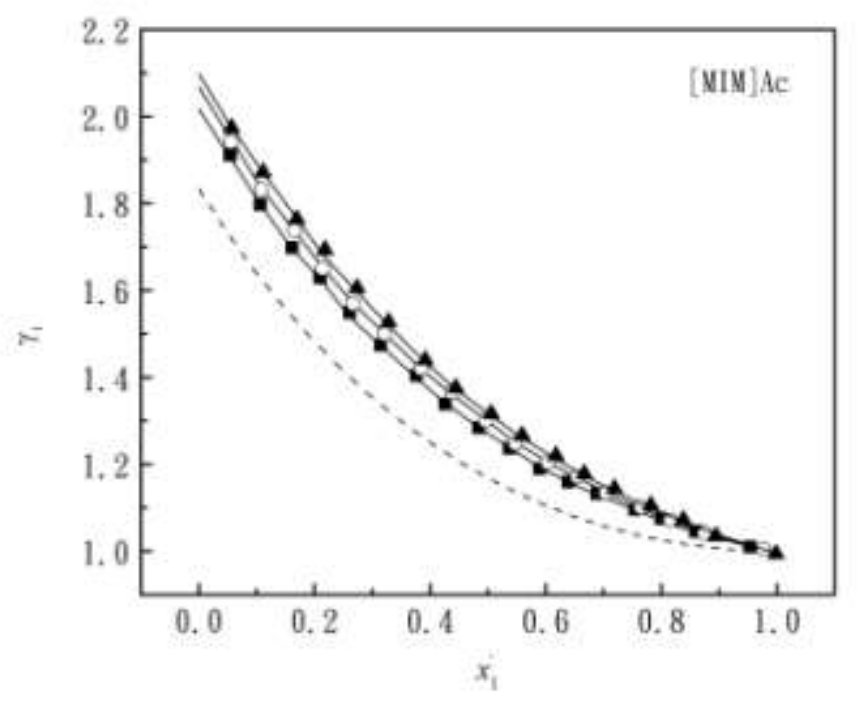

Fig.10. Activity coefficient of acetone $\gamma 1$ in relation with the mole fraction of acetone on an IL-free basis for the mixtures containing [MIM]Ac at 101.3kPa: dotted line, IL-free system; ., $\mathrm{x}_{3}=0.06 ; \circ, \mathrm{x}_{3}=0.08 ; \Delta, \mathrm{x}_{3}=\mathbf{0 . 1}$; solid line, correlated using NRTL model.

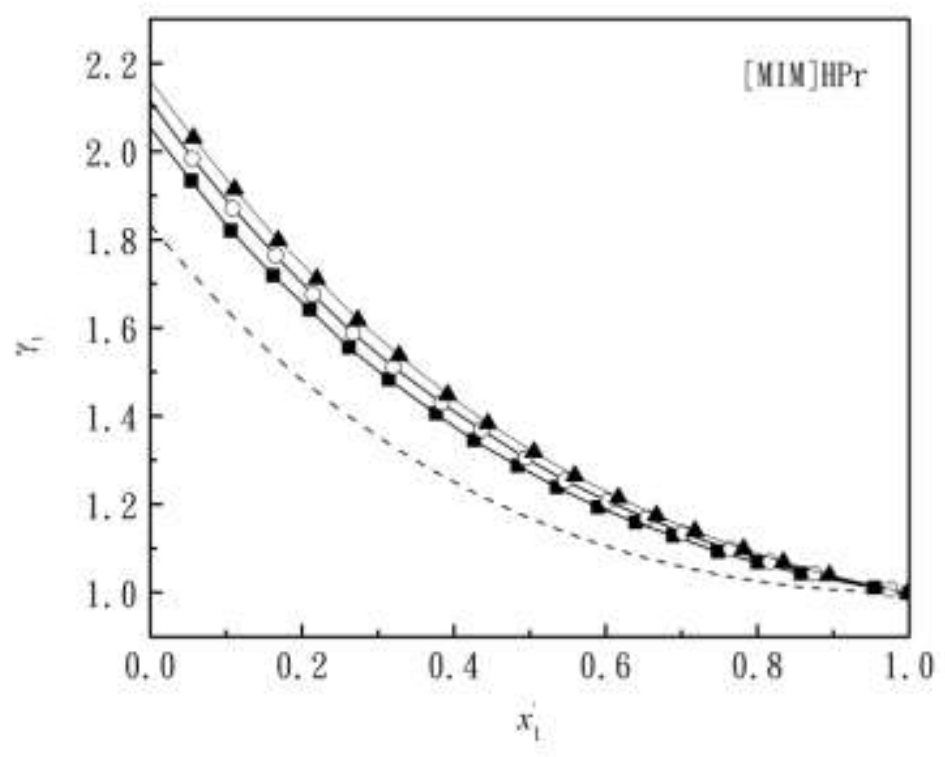

Fig.11. Activity coefficient of acetone $\gamma 1$ in relation with the mole fraction of acetone on an IL-free basis for the mixtures containing [MIM] HPr at 101.3kPa: dotted line, IL-free system; ., $x_{3}=0.06 ; \circ, x_{3}=0.08 ; \triangle, x_{3}=0.1 ; \quad$ solid line, correlated using NRTL model. 


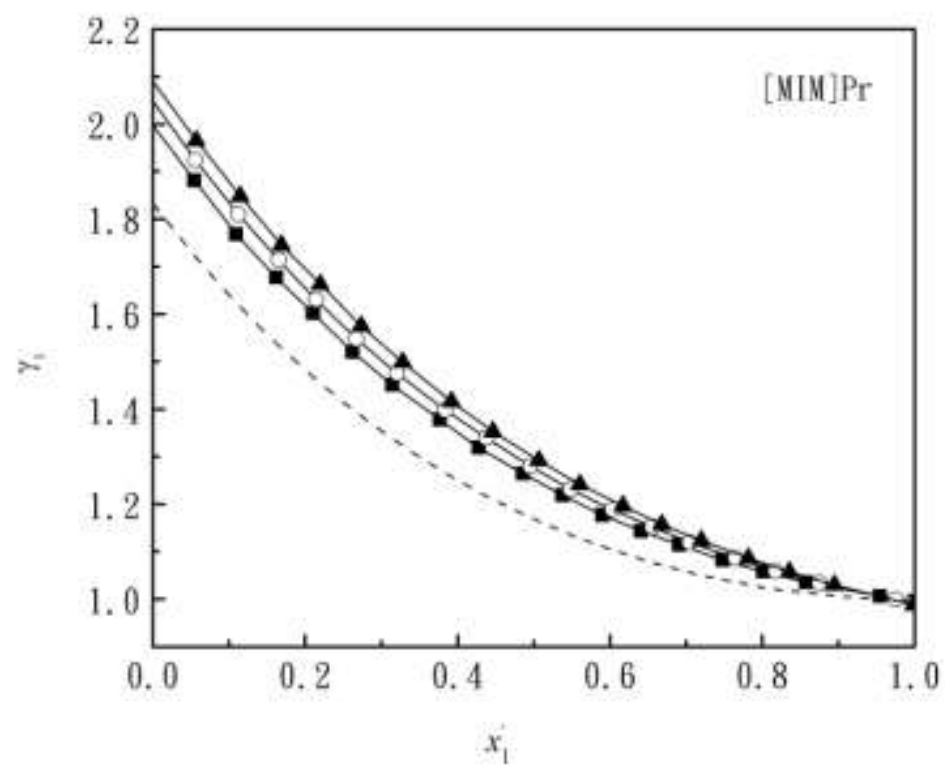

Fig.12. Activity coefficient of acetone $\gamma 1$ in relation with the mole fraction of acetone on an IL-free basis for the mixtures containing [MIM] Pr at 101.3kPa: dotted line, IL-free system; $\mathrm{a}, \mathrm{x}_{3}=0.06 ; \circ, \mathrm{x}_{3}=0.08 ; \Delta, \mathrm{x}_{3}=0.1$; solid line, correlated using NRTL model.

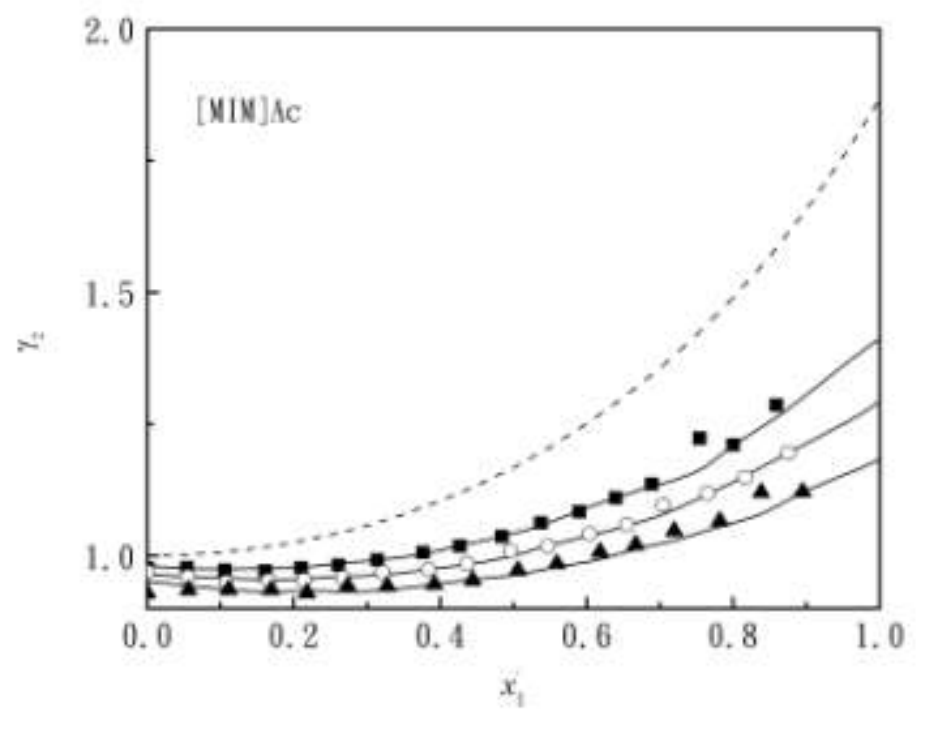

Fig.13. Activity coefficient of methanol $\gamma 2$ in relation with the mole fraction of acetone on an IL-free basis for the mixtures containing [MIM]Ac at 101.3kPa: dotted line, IL-free system; ., $x_{3}=0.06 ; \circ, x_{3}=0.08 ; \Delta, x_{3}=0.1 ;$ solid line, correlated using NRTL model. 


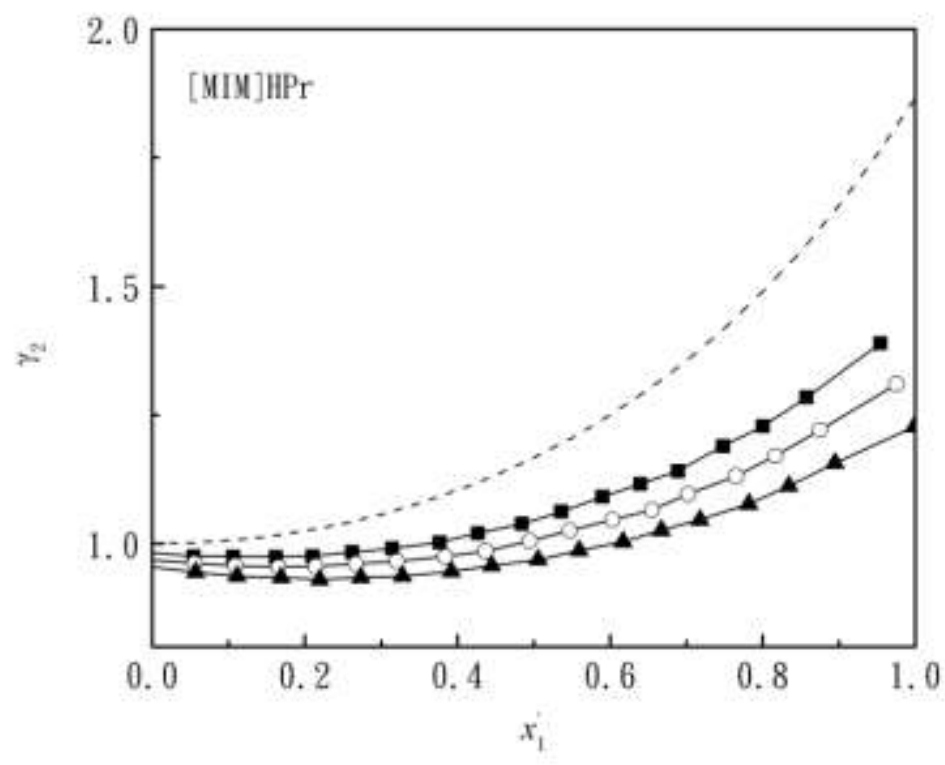

Fig.14. Activity coefficient of methanol $\gamma 2$ in relation with the mole fraction of acetone on an IL-free basis for the mixtures containing [MIM] HPr at 101.3kPa: dotted line, IL-free system; ., $\mathrm{x}_{3}=0.06 ; \circ, \mathrm{x}_{3}=0.08 ; \Delta, \mathrm{x}_{3}=0.1 ;$ solid line, correlated using NRTL model.

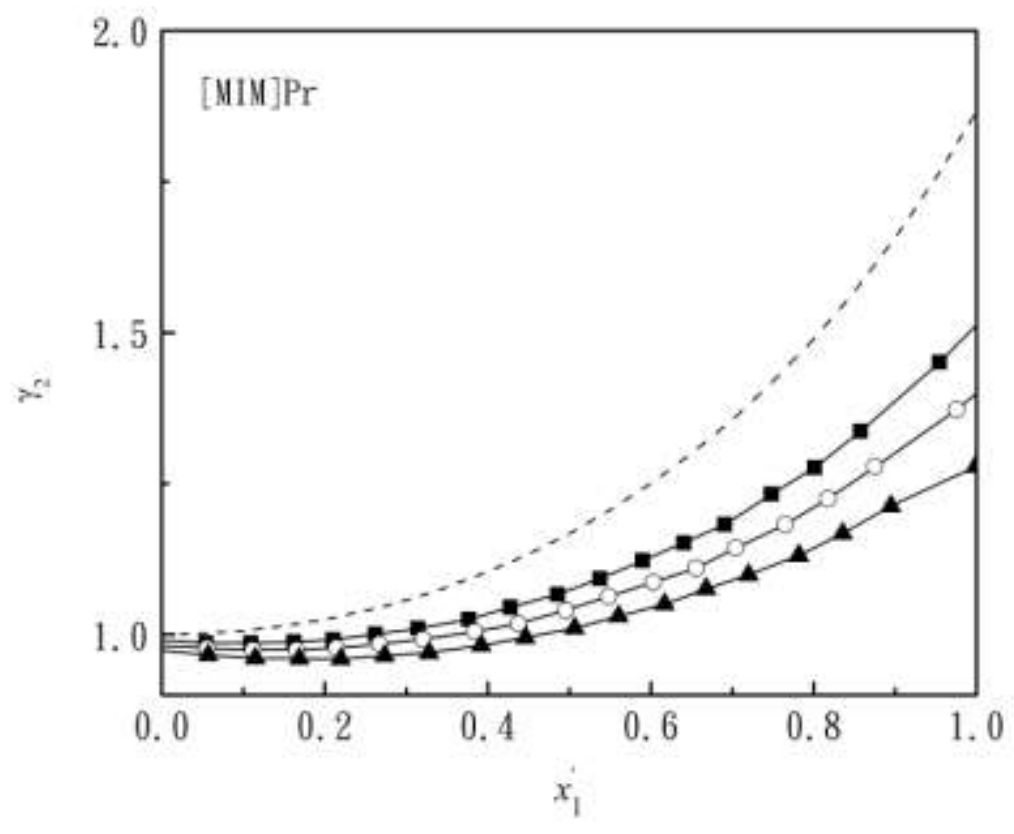

Fig.15. Activity coefficient of methanol $\gamma 2$ in relation with the mole fraction of acetone on an IL-free basis for the mixtures containing [MIM]Pr at 101.3kPa: dotted line, IL-free system; $\square, x_{3}=0.06 ; \circ, x_{3}=0.08 ; \Delta, x_{3}=0.1 ;$ solid line, correlated using NRTL model. 
The separation abilities of the three ILs for acetone and methanol should be attributed to the different intensities of the hydrogen bonds between them. The azeotropic phenomenon of methanol-acetone system is mainly due to the hydrogen bond between the hydroxyl group of methanol and the carbonyl group of acetone. The anions and imidazole rings of the three ILs can also form hydrogen bonds with hydroxyl group of methanol as hydrogen bonding acceptor and donator respectively, but the hydrogen bonds are stronger than that between methanol and acetone [22, 23]. Besides, it can be concluded from Figs.10-12 that all the three ILs have repulsive forces to acetone. Hence the addition of ILs can break the hydrogen bond between methanol and acetone. After the content of ILs is increased to a certain value, the azeotropic phenomenon of methanol-acetone system is eliminated.

According to the NRTL model, the separation abilities of the three ILs are $[\mathrm{MIM}] \mathrm{Ac}>[\mathrm{MIM}] \mathrm{HPr}>[\mathrm{MIM}] \mathrm{Pr}$. For $[\mathrm{MIM}] \mathrm{Pr}$ and $[\mathrm{MIM}] \mathrm{Ac}$, the hydrophobicity of $[\mathrm{MIM}] \operatorname{Pr}$ is stronger than that of $[\mathrm{MIM}] \mathrm{Ac}$ due to the longer alkyl chain length, so [MIM]Pr produces weaker hydrogen bond with methanol than [MIM]Ac[25].

The hydroxyl group of [MIM]HPr can form hydrogen bonds with the hydroxyl group of methanol as hydrogen bonding acceptor and donator, and with the carbonyl group of acetone as hydrogen bonding donator. The hydrogen bond between the hydroxyl group of [MIM]HPr and that of methanol is stronger than that between the hydroxyl group of [MIM]HPr and the carbonyl group of acetone. Hence, the hydroxyl group on $[\mathrm{MIM}] \mathrm{HPr}$ can enhance the relative volatility of acetone to methanol, leading to the better separation effect of [MIM]HPr than [MIM]Pr.

[MIM]HPr and [MIM]Ac show complex effects on mixture of acetone and methanol. At areas of higher methanol content, [MIM]HPr shows the larger relative volatility than [MIM]Ac due to the hydrogen bond between the hydroxyl group of [MIM]HPr and the hydroxyl group of methanol. At areas of higher acetone content, the methanol molecules are surrounded by acetone molecules. The hydrogen bond between the hydroxyl group of [MIM]HPr and the hydroxyl group of methanol is weakened by the hydrogen bond between acetone and [MIM]HPr with the increase of acetone content. As a result of the integrative effect, the minimum mole content of [MIM]HPr needed to eliminate the azeotropy is more than that of[MIM]Ac, showing the weaker separation ability than [MIM] Ac.

The minimum mole fractions of ILs with inorganic anions to totally break the azeotropy of acetone + methanol system are in the range of 0.022 to $0.079[12,13$, 19-23], so the separation ability of [MIM]Ac is comparable to that of ILs with inorganic anions. In addition, the periods of validity for the three ILs are all more than 9 months by the VLE and liquid chromatography measurements. On the other hand, [MIM]Ac is no pollution and corrosion of inorganic anions, such as $\mathrm{BF}_{4}^{-}, \mathrm{SO}_{4}{ }^{2-}$ and $\mathrm{Cl}^{-}$. From this standpoint, $[\mathrm{MIM}] \mathrm{Ac}$ is a potential entraner for the separation of acetone and methanol. 


\section{Conclusions}

In this work, the isobaric VLE data for acetone and methanol in the presence of [MIM]Ac, [MIM]Pr or [MIM]HPr were measured. A remarkable salting out effect is produced by the addition of ILs. The VLE data are well correlated using NRTL model. The salting out effects of the three ILs with organic anions are [MIM]Ac > $[\mathrm{MIM}] \mathrm{HPr}>[\mathrm{MIM}] \mathrm{Pr}$. The minimum mole content of [MIM]Ac needed to eliminate the azeotropy is 0.067 . The separation ability of [MIM]Ac is comparable to that of ILs with inorganic anions previously reported.

\begin{tabular}{|cl|}
\hline \multicolumn{2}{|c|}{ Nomenclature } \\
$\mathrm{x}_{i}$ & Mole fraction of solvent $i$ in the liquid phase \\
$\mathrm{y}_{i}$ & Mole fraction of solvent $i$ in the vapor phase \\
$\mathrm{T}$ & Equilibrium temperature \\
$\Delta \mathrm{g}_{i j}$ & Binary energy parameter of NRTL model \\
$y_{i}^{\text {eal }}$ & Mole fraction of solvent $i$ in the vapor phase calculated with the NRTL model \\
$\mathrm{P}$ & Total pressure in the equilibrium system \\
$P_{i}^{\circ}$ & Saturated vapor pressure of component $i$ at equilibrium temperature \\
& \\
& Greek letters \\
$\alpha_{12}$ & relative volatility of component 1 to component 2 \\
$\alpha_{i j}$ & non-randomness parameter of NRTL model \\
$\gamma_{\mathrm{i}}$ & Activity coefficient of component $i$ \\
$y_{i}^{\text {exp }}$ & The activity coefficient of component $i$ measured by experimental data \\
$\gamma_{i}^{\text {oal }}$ & The activity coefficient of component $i$ calculated with the NRTL model \\
\hline
\end{tabular}

\section{Acknowledgments}

This work is financially supported by the National Science Foundation of China (Project No. 21576166), Program for Liaoning Excellent Talents in University (LR2012013) and Liaoning Province Science Foundation of China (Project No. 2014020140).

\section{References}

[1] Z. Lei, C. Li, B. Chen, Extractive distillation: a review, Sep. Purif. Rev. 32 (2003) 121-213.

[2] Z. Lei, B. Chen, Z. Ding, Special Distillation Processes, Elsevier, Amsterdam, 2005.

[3] W.L. Luyben, Effect of solvent on controllability in extractive distillation, Ind. Eng. Chem. Res. 47 (2008) 4425-4439.

[4]Z. Lei, H. Wang, R. Zhou, Z. Duan, Influence of salt added to solvent on extractive distillation, Chem. Eng. J. 87 (2002) 149-156.

[5]W.L.Luyben, Comparison of Extractive Distillation and Pressure-Swing Distillation for 
Acetone-Methanol Separation, Ind.Eng. Chem. Res. 47 (2008) 2696-2707.

[6]M.Seiler, C. Jork, W. Schneider, W. Arlt, Ionic liquids and hyperbranched polymers promissing new classes of selective entrainers for extractive distillation, in: Proceedings of the International Conference on Distillation \& Absorption 2002; GVC-VID: Dusseldorf, 2002.

[7] A. Arce, H. Rodríguez, A. Soto, Purification of ethyl tert-butyl ether from its mixtures with ethanol by using an ionic liquid, Chem. Engi. J. 115 (2006) 219-223.

[8] L.-Z. Zhang, D.-S. Deng, J.-Z. Han, D.-X. Ji, J.-B. Ji, Isobaric Vapor-Liquid Equilibria for Water + 2-Propanol + 1-Butyl-3-methylimidazolium Tetrafluoroborate, J. Chem. Eng. Data 52 (2007) 199-205.

[9] Q. Li, F. Xing, Z. Lei, B. Wang, Q. Chang, Isobaric vapor-liquid equilibrium for isopropanol + water + 1-ethyl-3-methylimidazolium tetrafluoroborate, J. Chem. Eng. Data 53 (2008) 275-279.

[10] Q. Li, J. Zhang, Z. Lei, J. Zhu, F. Xing, Isobaric Vapor-Liquid Equilibrium for Ethyl Acetate + Ethanol + 1-Ethyl-3-methylimidazolium Tetrafluoroborate, J. Chem. Eng. Data 54 (2009) 193-197.

[11] D. Deng, R. Wang, L. Zhang, Y. Ge, J. Ji, Vapor-liquid equilibrium measurements and modeling for ternary system water + ethanol + 1-butyl-3-methylimidazolium acetate, Chin. J. Chem. Eng. 19 (2011) 703-708.

[12] A.V. Orchilles, P.J. Miguel, F.J. Llopis, E. Vercher, A. Martinez-Andreu, Influence of Some Ionic Liquids Containing the Trifluoromethanesulfonate Anion on the Vapor-Liquid Equilibria of the Acetone + Methanol System, J. Chem. Eng. Data, 56 (2011) 4430-4435.

[13]A.V. Orchilles, P.J. Miguel, V. Gonzalez-Alfaro, E. Vercher, A. Martinez-Andreu, 1-Ethyl-3-methylimidazolium Dicyanamide as a Very Efficient Entrainer for the Extractive Distillation of the Acetone + Methanol System, J. Chem. Eng. Data 57 (2012) 394-399.

[14]Z.Zhang, A.Hu, T.Zhang, Q.Zhang, M.Sun, D. Sun and W. Li, Separation of methyl acetate + methanol azeotropic mixture using ionic liquids as entrainers, Fluid Phase Equilib. 401 (2015) $1-8$.

[15]Z.Zhang, F.Pan, Q.Zhang, T.Zhang, L.Zhang, K.Fang and W. Li, Isobaric Vapor-Liquid Equilibria for Ethyl Acetate + Methanol + Ionic Liquids Ternary Systems at $101.3 \mathrm{kPa}$, J. Chem. Eng. Data 61 (2016) 772-779.

[16]Z.Zhang, A.Hu, T.Zhang, Q.Zhang, Z.Yang and W. Li, Isobaric vapor-liquid equilibrium for methyl acetate + methanol system containing different ionic liquids at $101.3 \mathrm{kPa}$, Fluid Phase Equilib. 408 (2016) 20-26.

[17] Z.Zhang, L.Zhang, Q.Zhang, D.Sun, F.Pan, S.Dai and W. Li, Separation of 2-propanol and water azeotropic system using ionic liquids as entrainers, Fluid Phase Equilib. 412 (2016) 94-100.

[18]W.Arlt, M.Seiler, C.Jork, T.Schneider, Ionic liquids as selective additive for the separation of close-boiling or azeotropic mixtures. WO2002/074718 A2, 2002-09-26.

[19] A.V. Orchillés, P.J. Miguel, E. Vercher, A. Martínez-Andreu, Ionic Liquids as Entrainers in Extractive Distillation: Isobaric Vapor-Liquid Equilibria for Acetone + Methanol + 1-Ethyl-3-methylimidazolium Trifluoromethanesulfonate, J. Chem. Eng. Data 52 (2007) 141-147.

[20] X. Chen, B. Yang, A.A. Abdeltawab, S.S. Al-Deyab, G. Yu, X. Yong, Isobaric Vapor-Liquid Equilibrium for Acetone + Methanol + Phosphate Ionic Liquids, J. Chem. Eng. Data 60 (2015) 612-620. 
[21] H. Matsuda, V. Liebert, K. Tochigi, J. Gmehling, Influence of sulfate-based anion ionic liquids on the separation factor of the binary azeotropic system acetone+methanol, Fluid Phase Equilib. 340 (2013) 27-30.

[22] W. Li, D. Sun, T. Zhang, S. Dai, F. Pan, Z. Zhang, Separation of acetone and methanol azeotropic system using ionic liquid as entrainer, Fluid Phase Equilib. 383 (2014) 182-187.

[23] W. Li, D. Sun, T.Zhang, Y. Huang, L. Zhang, and Z. Zhang, Phase equilibrium study of binary and ternary mixtures of Ionic liquids + acetone + methanol, J. Chem. Eng. Data 59(2014) 3975-3981

[24]W. Hunsmann, Evaporation equilibrium of formic acid-acetic acid and carbon tetrachloride-perchloroethylene mixtures, Chem.Ing.Tech., 39 (1967) 1142-1145.

[25] Z.Zhang, Q.Zhang, Q.Q.Zhang, T.Zhang, and W. Li, Isobaric Vapor-Liquid Equilibrium of tert-Butyl Alcohol + water + Triethanolamine-Based Ionic Liquid Ternary Systems at 101.3kPa, J. Chem. Eng. Data 60 (2015) 2018-2027. 